\title{
Long-term and short-term prognostic value of the prognostic nutritional index in cancer: a narrative review
}

\author{
Li Yan', Tomoki Nakamura ${ }^{2}$, Andrea Casadei-Gardini ${ }^{3}$, Gema Bruixola ${ }^{4}$, Yuan-Lan Huang ${ }^{5}$, Zhi-De Hu ${ }^{6}$ \\ ${ }^{1}$ Department of Respiratory and Critical Care Medicine, the Affiliated Hospital of Inner Mongolia Medical University, Hohhot, China; ${ }^{2}$ Department \\ of Orthopaedic Surgery, Mie University Graduate School of Medicine, Tsu-city, Mie, Japan; ${ }^{3}$ Università Vita-Salute, San Raffaele Hospital-IRCCS, \\ Milano, Italy; ${ }^{4}$ Department of Medical Oncology, Biomedical Research Institute INCLIVA, University of Valencia, Valencia, Spain; ${ }^{5}$ Department of \\ Special Food and Equipment, Naval Special Medical Center, the Naval Military Medical University, Shanghai, China; ${ }^{6}$ Department of Laboratory \\ Medicine, the Affiliated Hospital of Inner Mongolia Medical University, Hohhot, China \\ Contributions: (I) Conception and design: All authors; (II) Administrative support: Yan L; (III) Provision of study materials or patients: All authors; (IV) \\ Collection and assembly of data: ZD Hu; (V) Data analysis and interpretation: All authors; (VI) Manuscript writing: All authors; (VII) Final approval \\ of manuscript: All authors. \\ Correspondence to: Yuan-Lan Huang, PhD. Department of Special Food and Equipment, Naval Special Medical Center, the Naval Military Medical \\ University, Shanghai 200433, China. Email: huang_yuanlan@163.com; Zhi-De Hu, PhD. Department of Laboratory Medicine, the Affiliated \\ Hospital of Inner Mongolia Medical University, Hohhot 010010, China. Email: hzdlj81@163.com.
}

\begin{abstract}
Objective: To perform a narrative review of the prognostic value of prognostic nutritional index (PNI) in cancers.

Background: Prognostic estimation greatly determines the treatment approach in various cancers. The PNI, calculated using the serum albumin level and total lymphocyte count, is a useful indicator to assess nutritional and immunological conditions. The PNI represents a low-cost, easy-to-perform, noninvasive, rapid, and standardized tool for estimating the prognosis of cancer. Many studies have aimed to clarify the prognostic value of PNI for various types of cancer.

Methods: We summarize the studies, particularly the systematic reviews and meta-analyses, that have examined the prognostic value of PNI in common cancers.

Conclusions: The relevant studies indicate that low PNI is an independent prognostic factor for decreasing overall survival in many types of cancers. Disease-free survival and progression-free survival were also associated with PNI in some types of cancer including lung cancer and renal cell carcinoma. Therefore, we suggest that the measurement of PNI is a useful method to identify cancer patients that have a worse prognosis and that the treatment strategy for these patients be adjusted accordingly. We hypothesize that maintaining good nutritional status during treatment may improve outcomes of various cancers.
\end{abstract}

Keywords: Prognostic nutrition index (PNI); cancer; prognosis; review

Submitted Jul 08, 2021. Accepted for publication Oct 02, 2021.

doi: $10.21037 / \mathrm{atm}-21-4528$

View this article at: https://dx.doi.org/10.21037/atm-21-4528

\section{Introduction}

Prognostic estimation is crucial for cancer management because it can significantly inform the selection of the treatment approach. The conventional prognostic estimation tools include medical imaging, pathological methods, demographic factors, and laboratory testing. However, none of these tools can accurately predict the prognosis of cancer patients when used alone. They each have distinct limitations, including invasiveness, high cost, requirement of special training, and subjectivity. The ideal prognostic estimation tool should be noninvasive, easy to use, low cost, and standardized. Some tools can meet these requirements, such as the prognostic nutritional index (PNI) (1) and controlling nutritional status (CONUT) 
score (2). In this narrative review, we focus on the prognostic value of $\mathrm{PNI}$ in cancer.

PNI was first proposed by Buzby in 1980 (1). The formula for calculating the PNI was based on 4 factors: albumin, triceps skinfold, transferrin, and skin test reactivity. Partly due to the subjectivity involved in triceps skinfold and skin test reactivity, the clinical application of PNI was limited. In 1984, Onodera modified the formula to the following: PNI=albumin $(\mathrm{g} / \mathrm{L})+5 \times$ absolute lymphocyte count $\left(10^{9} / \mathrm{L}\right)(3)$. Compared to the initial formula, the modified formula has some advantages, such as being low cost and easy to perform, and having a short turnaround time and standardized design. Because albumin and absolute lymphocyte count are greatly affected by the nutritional status of individuals and because cancer patients are often malnourished, PNI is usually used to estimate the prognosis of cancer patients.

In 2014, a systematic review and meta-analysis investigated the prognostic value of PNI in various types of cancers (4). This meta-analysis included 14 studies with 3,413 cancer patients and revealed that the pooled hazard ratio (HR) of low PNI for overall survival (OS) and cancerspecific survival (CSS) was 1.80 [95\% confidence interval (CI): 1.59-2.04] and 2.45 (95\% CI: 1.31-4.58), respectively. These results indicate that low PNI is a risk factor for a worse prognosis in cancer patients. Furthermore, a subgroup analysis indicated that the type of cancer, region, surgery type, cutoff value, and sample size did not significantly affect the pooled HR. In addition, low PNI was associated with postoperative complications with a pooled odds ratio (OR) of 2.45 (95\% CI: 1.31-4.58). Subsequently, mounting studies have been performed to investigate the prognostic value of PNI in various types of cancer. For some types of cancer, systematic reviews and meta-analyses have been performed to determine the prognostic value of PNI.

Because the results from individual cohort studies are heterogeneous, meta-analyses are needed to pool available studies. It is generally accepted that the meta-analysis is more convincing than the individual study. In this narrative review, we summarize the studies, particularly the systematic reviews and meta-analyses, that have examined the prognostic value of PNI in common cancers.

We present the following article in accordance with the Narrative Review reporting checklist (available at https:// dx.doi.org/10.21037/atm-21-4528).

\section{Respiratory system neoplasms}

\section{Lung cancer}

The most common respiratory system neoplasm is lung cancer (LC). In 2018, 2 meta-analyses investigated the prognostic value of PNI in LC (5,6). In 1 study, low PNI was associated with lower OS with an HR of 1.72 (95\% CI: 1.43-2.06) (5). A subgroup analysis revealed that low PNI remained a risk factor for low OS across some subgroups (i.e., region, sample size, tumor stage, histology, and study quality) (5). These findings were validated by subsequent updated meta-analyses, which searched additional databases and included more studies for both small cell lung cancer (SCLC) and non-small cell lung cancer (NSCLC) (6-8).

In addition to OS, the associations between PNI and disease-free survival (DFS) or recurrence-free survival (RFS) and progression-free survival (PFS) were also investigated in both SCLC and NSCLC (6-8). In 2 meta-analyses, although the number of included studies was small, a significant association between low PNI and worse RFS and PFS in SCLC (8) and NSCLC $(6,7)$ was observed. In addition, a study with 80 late-stage lung cancer patients (stage III and IV) revealed the low PNI associated with low quality of life, which is measured by the FACT-L questionnaire (9). Notably, most of the available studies in these meta-analyses were from East Asia, particularly China and Japan (5-8). Therefore, more evidence from Europe and America is needed to validate the findings of these meta-analyses.

\section{Other respiratory system neoplasms}

Only 2 studies have investigated the prognostic value of PNI in malignant pleural mesothelioma (MPM) $(10,11)$, with both concluding that low PNI is a risk factor for worse OS. PNI was found to be associated with age, smoking, and weight loss (11).

\section{Head and neck tumors}

\section{Nasopharyngeal carcinoma}

Thus far, 3 meta-analyses have investigated the prognostic value of PNI in nasopharyngeal carcinoma (12-14). Each of these meta-analyses included approximately 10 studies and revealed that low PNI was a risk factor for worse OS, 
PFS, and distant metastasis-free survival (DMFS) (12-14). However, the results regarding locoregional failure-free survival (LRFFS) were inconsistent. In 1 meta-analysis, low PNI was associated with worse LRFSS (12), while another meta-analysis failed to find an association between low PNI and worse LRFSS (13). The possible reasons for the inconsistency remain unclear. Moreover, a subgroup analysis reported that the HR of PNI for OS and DMFS remained statistically significant across all treatment regimens (12).

The correlations between PNI and clinical characteristics of nasopharyngeal carcinoma have also been investigated. Low PNI has been associated with female sex $(13,14)$, older age $(13,14)$, advanced stage $(13,14)$, high alanine transaminase (ALT), and high white blood cell (WBC) count (13).

\section{Head and neck squamous cell carcinoma (HNSCC)}

HNSCC includes some types of cancer that originate from different regions, such as the oropharynx, hypopharynx, oral cavity, and larynx. A retrospective study published in 2018 indicated that PNI is an independent risk factor for OS in patients with HNSCC ( $\mathrm{n}=145)$ (15). Patients with PNI $<45$ have approximately 3 times higher of risk for death than patients with PNI $>45$. Another retrospective study $(\mathrm{n}=101)$ revealed that low PNI $(<40)$ is associated with high risk of severe adverse events in HNSCC patients treated with radiotherapy (16).

A retrospective study enrolled 103 patients with oral squamous cell carcinoma (OSCC) to analyze the association between PNI and disease-specific survival (DSS) (17). Low PNI was associated with decreased DSS with an HR of 0.86 (95\% CI: 0.76-0.97) (17). Thus far, no systematic reviews or meta-analyses have been performed to investigate the prognostic value of PNI in HNSCC.

\section{Digestive tract cancer}

The first meta-analysis regarding PNI and digestive system carcinomas was published in 2016 (18). A total of 23 studies, covering various types of digestive system carcinomas (e.g., gastric cancer, esophageal carcinoma, and colorectal carcinoma), were included. The PNI's pooled HR was 1.83 (95\% CI: $1.62-2.07)$ for OS and 1.85 (95\% CI: 1.19-2.89) for DFS. The authors failed to find a significant association between PNI and CSS perhaps because only 2 studies investigated the association between PNI and CSS and the sample size is small. A subgroup analysis revealed that the region, sample size, cutoff value, and cancer type did not significantly affect the prognostic value of PNI. In addition, they also found that low PNI was associated with high postoperative complications (HR $=2.31 ; 95 \% \mathrm{CI}$ : 1.63-3.28). Subsequently, several studies have investigated the prognostic value of PNI in specific digestive system carcinomas. The following sections summarize the findings of the meta-analyses investigating the prognostic value of $\mathrm{PNI}$ in various digestive tract cancers.

\section{Esophageal cancer (EC)}

Three systematic reviews and meta-analyses have examined the prognostic value of PNI in EC, irrespective of histological type and treatment (19-21). Most of the included studies were identical, and thus the findings are similar. Each of these meta-analyses found that low PNI was a risk factor for worse OS and CSS. One of the metaanalyses investigated the association between PNI and RFS and did not find a significant association between PNI and RFS (HR =1.63; 95\% CI: 0.90-2.95) (20). Two metaanalyses investigated the correlation between PNI and the clinicopathological features of EC $(19,20)$. The authors found that low PNI was correlated with male sex $(19,20)$, old age (19), positive lymph node status (20), advanced T stage $(19,20)$, and body mass index (BMI) (20), but not metastasis $(19)$ or the differentiation grade $(19,20)$ of EC. Meanwhile, another study found that patients with low PNI were at high risk of postoperative complications (21). In a subgroup analysis, the pooled HR was found to be statistically significant across different groups (e.g., country, year of publication, sample size, cutoff value, and quality scores) $(19,20)$. Notably, 1 meta-analysis (20) performed a sensitivity analysis and revealed that the results of the metaanalysis were greatly affected by the study performed by Matsumoto et al. (22). In addition, most of the included studies in these 3 meta-analyses were from China and Japan and used a retrospective design. Therefore, prospective studies with European, Africa, and American patients are needed to validate the findings of these 2 meta-analyses.

Pathologically, EC is categorized into squamous cell carcinoma (ESCC) and adenocarcinoma (EADC). The above-mentioned meta-analyses did not perform subgroup analysis to investigate the prognostic value of PNI in ESCC or EADC. In 2020, a systematic review included 9 studies in its investigation into the association between ESCC prognosis and PNI (23). The pooled HRs of low PNI for OS and PFS were 1.42 (95\% CI: $1.20-1.68)$ and 1.88 (95\% 
CI: 1.21-2.93), respectively, but low PNI was not associated with CSS (HR $=1.95 ; 95 \%$ CI: 0.54-6.98; $\mathrm{P}=0.31)$. This negative finding may be attributed to the fact that only 2 studies concerning CSS were included and had considerable heterogeneity (24,25). PNI in ESCC patients was not associated with age, gender, depth of tumor, differential grade, tumor-node-metastasis (TNM) stage, or lymph node metastasis.

\section{Gastric cancer}

In 2016, 2 meta-analyses investigated the prognostic value of PNI in gastric cancer (GC) (26,27). Both meta-analyses found that low PNI was associated with OS $(26,27)$. Subgroup analysis revealed that the association between PNI and prognosis of GC was not affected by treatment or the cutoff used (26). These findings were validated by an updated meta-analysis published in 2019 (28). By including studies investigating the prognostic value of PNI in GC patients following gastrectomy, the authors found that the pooled HR (95\% CI) was 1.81 (1.56-2.09) for OS, 1.61 (1.24-2.10) for CSS, and $1.82(1.20-2.77)$ for RFS. In addition, they found that low PNI was a risk factor for the occurrence of postoperative complications and increased mortality $(27,28)$. Meanwhile, low PNI was associated with the clinical and pathological characteristics of GS, such as older age $(27,28)$, bigger tumor size $(27,28)$, tumor stage $(27,28)$, lower BMI (28), deep tumor depth (28), and positive vessel invasion $(27,28)$. Because all the included studies were from East Asia, caution should be taken in extending the findings of this study to areas other than East Asia.

\section{Colorectal cancer (CRC)}

In 2016, Yang et al. performed a retrospective study and meta-analysis to investigate the association between PNI and the outcome of CRC (29). In the meta-analysis, 10 studies with 3,582 CRC patients were included. The pooled HR was 1.97 (95\% CI: 1.54-2.53) for OS and 1.48 (95\% CI: 1.19-1.85) for CSS. In a subsequent subgroup analysis, region, surgery, TNM stage, cutoff value, sample size, and study quality did not affect the association between PNI and CRC outcome. The findings of this meta-analysis were validated by an updated meta-analysis published in 2019 (30). The updated meta-analysis included 10 studies to investigate the prognostic value of PNI in CRC patients treated with surgery. In addition to observing that low PNI was associated with worse OS, DFS, and CSS in all groups and subgroups, the study also found low PNI to be associated with postoperative complications (30).

\section{Hepatobiliary and pancreatic cancers}

\section{Hepatocellular carcinoma (HCC)}

Three meta-analyses have investigated the prognostic value of PNI in HCC (31-33). Each of these meta-analyses concluded that low PNI was associated with worse OS and DFS (31-33). The ORs of PNI for 1-, 3-, and 5-year OS were 2.91 (95\% CI: 2.30-3.70), 4.05 (95\% CI: 3.27-5.03), and 3.65 (95\% CI: 2.96-4.50), respectively (31). In a subgroup analysis, region and gender did not affect the association between the PNI and OS of HCC (32). Notably, in patients treated with surgical resection or transcatheter arterial chemoembolization (TACE), the pooled HRs for OS remained statistically significant (32). This finding was validated by another meta-analysis in which only studies with curative hepatectomy-treated patients were included (33). In this meta-analysis, 7 studies were included, and the pooled HR for OS and RFS were 2.27 (95\% CI: $1.03-4.07)$ and 1.68 (95\% CI: 1.45-1.94), respectively (33). In addition, PNI was associated with serum alpha-fetoprotein (AFP), tumor size, and TNM stage, but not with tumor number or liver cirrhosis (31).

\section{Pancreatic cancer (PC)}

Thus far, 2 meta-analyses have examined the prognostic value of PNI in PC $(34,35)$. Although the literature search strategy and database differed between the 2 meta-analyses, the majority of included studies overlapped. Both metaanalyses reported low PNI to be associated with worse OS, with an HR around $1.5(34,35)$. Both performed subgroup analyses, which indicated low PNI to be associated with OS in the subgroups of treatment, stage, sample size, cutoff value, and study quality $(34,35)$. Notably, in a sensitivity analysis, the pooled HR was greatly affected by the study performed by Yamada et al. (36). In addition, most available studies were retrospective in design and from East Asia, which suggests that the findings of these meta-analyses need to be validated by prospective studies from areas outside of East Asia. 


\section{Biliary tract cancer}

In 2020, a systematic review and meta-analysis included 7 studies, which indicated low PNI to be associated with worse OS (HR $=1.65$; 95\% CI: 1.42-1.93) (37). The pooled HR for OS remained statistically significant in all subgroup analyses (i.e., treatment, ethnicity, and tumor differentiation). The authors also investigated the correlation between PNI and clinical characteristics and found low PNI to be associated with poor differentiation and higher $\mathrm{T}$ stage, but not gender or $\mathrm{N}$ stage.

\section{Urinary cancers}

The first systematic review and meta-analysis concerning urinary cancers were published in 2018 (38). In this review, 12 studies were deemed eligible for meta-analysis, including 6 on renal cell cancer (RCC), 3 on bladder cancer (BC), 2 on upper-tract urothelial carcinoma (UTUC), and 1 on prostate cancer. Low PNI was associated with worse OS, DFS/RFS/PFS, and CSS. Subgroup analyses revealed that the pooled HRs of PNI for OS, DFS/RFS/PFS, and CSS were not affected by the type of cancer or treatment approach (surgery or target therapy). The findings for RCC in this meta-analysis were validated by an updated meta-analysis published in 2021 (39). In the updated metaanalysis, 12 studies with 7,391 subjects were included. The pooled HR for OS was 1.97 (95\% CI: 1.60-2.43) while that for CSS and PFS was 1.78 (95\% CI: 1.23-2.57) and 1.94 (95\% CI: 1.62-2.31), respectively. Low PNI was associated with older age, less proportion of clear cell histology, advanced $\mathrm{T}$ stage, and higher Fuhrman grade. Notably, all included studies were retrospective in design; therefore, studies with a prospective design are needed to validate these findings.

In the meta-analysis published in 2018 (38), only 3 studies on $\mathrm{BC}$ were included. We noted that many studies concerning $\mathrm{PNI}$ and $\mathrm{BC}$ prognosis have been published since 2018 (40-42); therefore, an updated meta-analysis is needed to assess the prognostic value of PNI in BC as well as in prostate cancer $(40,43)$.

\section{Gynecological cancers}

In 2019, a systematic review and meta-analysis investigated the prognostic value of PNI for gynecological cancer (44). The review included 9 studies with 2,373 patients, with 4 focusing on ovarian cancer (OC), 1 on all gynecological cancers, and 4 on cervical cancer (CC). The meta-analysis revealed that low PNI was a risk factor for worse OS and PFS. For OC, the pooled HR for OS and PFS was 1.28 (0.73-2.26) and $1.82(1.41-2.36)$, respectively. These findings were validated by a recently published metaanalysis (45). For CC, the pooled HR for OS and PFS was $2.96(1.87-4.70)$ and 2.54 (1.41-4.58), respectively. Residual disease, ascites, and chemosensitivity were correlated with PNI (44).

To date, only 1 retrospective study has investigated the prognostic value of PNI in stage IVB endometrial cancer (46). Although low PNI was associated with decreased OS in univariate analysis, no statistical significance was found in the multivariable analysis. Considering that the sample size in this study was small $(n=32)$, further studies with larger samples are needed to validate the findings of this study. Moreover, no studies have investigated the prognostic value of PNI in choriocarcinoma or fallopian tube neoplasms.

\section{Central nervous system tumors}

In 2020, a systematic review and meta-analysis investigated the prognostic value of albumin, albumin-to-globulin ratio (AGR), and PNI for glioma (47). In this review, 9 of the 11 included studies investigated the association between PNI and OS in patients with glioma. The pooled HR of PNI for OS was not statistically significant (HR $=0.83$; 95\% CI: $0.60-1.16$ ); however, a subgroup analysis indicated PNI to be a protective factor for patients with high-grade gliomas (HR $=0.56$; 95\% CI: 0.43-0.73). In addition, those studies with a small sample size $(\mathrm{n}<200)$, defined cutoff value, or which performed multivariate analysis, reported elevated PNI to be associated with better OS. To the best of our knowledge, no study has yet investigated the prognostic value of PNI in meningioma, hypophyseal, or angioreticulocytoma.

\section{Breast cancer}

Some retrospective studies have investigated the prognostic value of PNI in various types of breast cancer (48-52). The target population in these studies varied and included patients who underwent surgery (48), those with triplenegative breast cancer (49), those with locally advanced 
breast cancer (50), those treated with eribulin (51), and those with $\mathrm{T} 1-2 \mathrm{~N} 1$ breast cancer treated with radiotherapy (52). The end point consisted of OS $(48,49)$, PFS (51,52), DFS $(49,50)$, and pathological complete response (pCR) (50). Each of these studies found that low PNI was associated with worse prognosis (48-52). The prognostic value of PNI was higher in premenopausal negative ER patients (50), while low PNI was associated with several clinical characteristics, including older age $(48,49)$, menopausal status (49), advanced T stage (49), advanced $\mathrm{N}$ stage (49), human epidermal growth factor receptor 2 (HER2) status (52), neutrophil-lymphocyte ratio (NLR) (52), monocyte-lymphocyte ratio (MLR) (52), and Ki67 status (52).

Changes in PNI after neoadjuvant chemotherapy were found also to be associated with worse prognosis. In a retrospective study with 191 patients, PNI before and after neoadjuvant chemotherapy was assessed (53). Although PNI before neoadjuvant chemotherapy was not associated with DFS, a high decrease of PNI during neoadjuvant chemotherapy was found to be a risk factor for decreased DFS. Subgroup analysis revealed that the prognostic value of changed PNI after neoadjuvant chemotherapy was significant in estrogen receptor (ER)-positive and HER2negative patients but not in ER-negative or HER2-positive patients.

\section{Thyroid cancer}

Two retrospective studies with a small sample size investigated the prognostic value of PNI in medullary thyroid carcinoma (54) and low-risk differentiated thyroid carcinoma (55). One study found that low PNI was associated with decreased RFS in univariate analysis; however, the multivariate analysis indicated no significant association between PNI and RFS (54). Regarding low-risk differentiated thyroid carcinoma, one study found there to be no significant association between PNI and RFS, either in univariate or multivariate analysis (55). In a multicenter retrospective study with 1,873 papillary thyroid carcinoma patients, low PNI was shown to be an independent risk factor for low RFS in multivariate analysis (56). Thus far, no meta-analysis has investigated the prognostic value of PNI in both breast and thyroid cancer.

\section{Hematologic malignancies}

Some studies have investigated the prognostic value of PNI in lymphomas, including follicular lymphoma (FL) (57), extranodal natural killer/T cell lymphoma (ENKTL), nasal type $(58,59)$, classic Hodgkin lymphoma (cHL) $(60,61)$, and diffuse large B cell lymphoma (DLBCL) (62). PNI was found to be associated with worse OS and/or PFS in ENKTL $(58,59)$ and FL $(57)$. However, the results from studies concerning cHL varied. One study of 122 patients with classic cHL reported that low PNI was associated with worse OS and PFS in univariate analysis; however, low PNI lost its significance in the multivariate analysis (61). In another study with large sample size $(\mathrm{n}=1,012)$, low PNI was an independent risk factor for OS and PFS in cHL (60).

A systematic review and meta-analysis published in 2020 investigated the prognostic value of PNI for DLBCL and included 7 studies (63). The pooled HR (95\% CI) was 2.14 (1.66-2.75) for OS and 1.75 (1.36-2.25) for PFS. However, 6 of the 7 included studies were from Asia, and thus more data from European and American patients are needed to validate the findings of PNI and the prognosis of DLBCL.

\section{Soft tissue sarcoma}

One retrospective study investigated the prognostic value of PNI in 100 patients with soft tissue sarcoma (64). The PNI was significantly inversely associated with tumor size and grade. In the multivariate analysis, PNI was a significant prognostic marker for CSS and event-free survival.

\section{Conclusions}

A number of studies have investigated the prognostic value of PNI in various types of cancer, and the evidence of the related meta-analyses are summarized in Table 1 . Generally, low PNI has been found to be associated with worse prognosis in various types of cancer, although there is no normal value established for PNI, and the molecular mechanisms underlying the prognostic effect of PNI on cancers remains unknown. Thus, maintaining a good nutritional status during treatment may improve outcomes in patients with cancer. PNI associates with some clinicopathological characteristics in many cancers; therefore, confounding factors should be adequately controlled or adjusted in future studies. In addition, most of the examined studies were performed in East Asia and had a retrospective design; therefore, prospective studies in African, American, and European populations are necessary to confirm the prognostic value of PNI in cancer. 
Table 1 Prognostic value of the prognostic nutritional index in cancers: evidence from meta-analyses

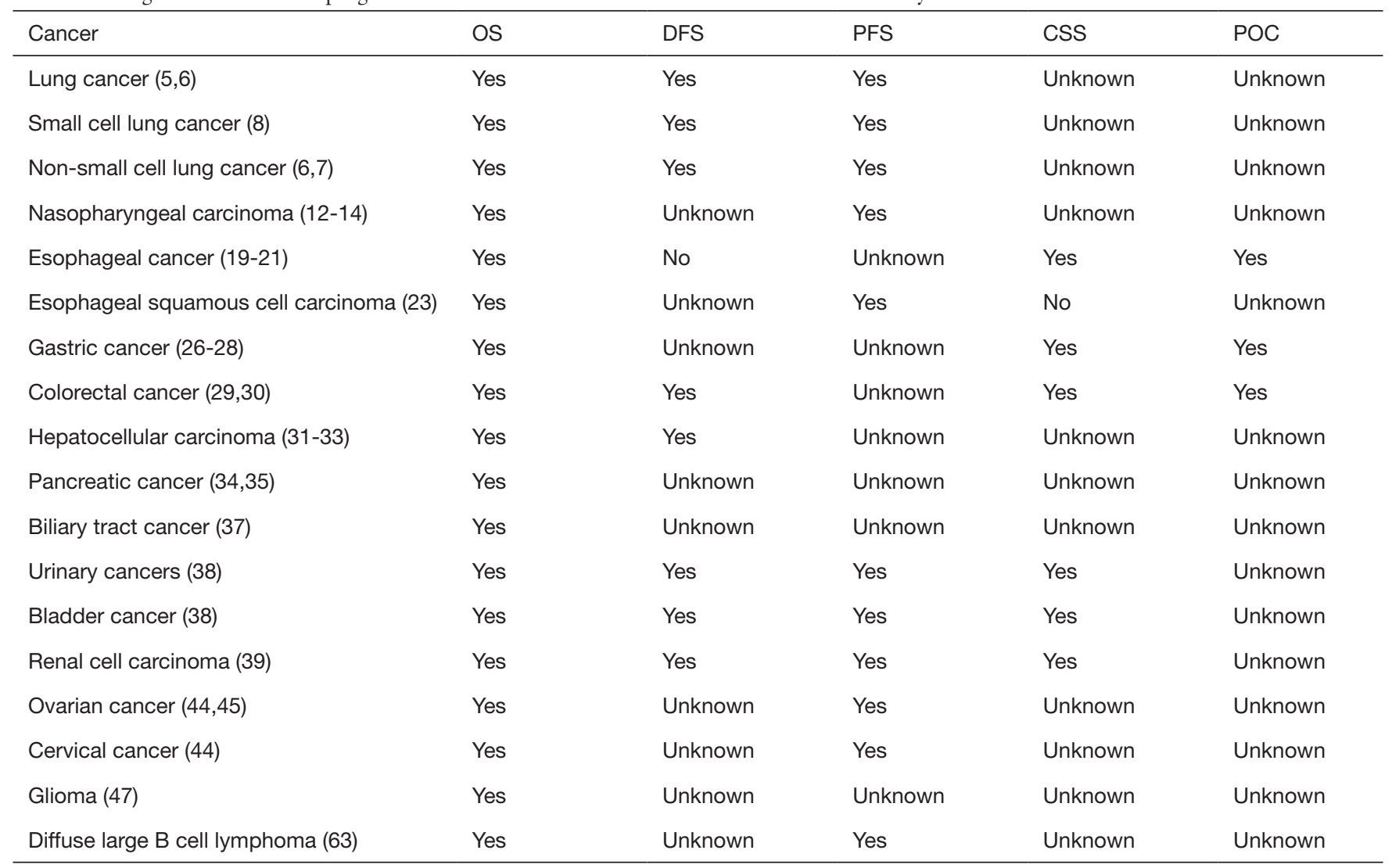

OS, overall survival; PFS, progression-free survival; DFS, disease-free survival; CSS, cancer-specific survival; POC, postoperative complication.

\section{Acknowledgments}

We thank Hideaki Shimada (Department of Gastroenterological Surgery and Clinical Oncology, Graduate School of Medicine, Toho University, 6-11-1 Omori-nishi, Ota-ku, Tokyo, 142-8541, Japan) for his valuable suggestions. The authors also appreciate the academic support from the AME Cancer Collaborative Group.

Funding: This work is supported by the National Natural Science Foundation of China (No. 81860501) and the Natural and Science Foundation of Inner Mongolia Autonomous Region for Distinguished Young Scholars (No. 2020JQ07).

\section{Footnote}

Reporting Checklist: The authors have completed the Narrative Review reporting checklist. Available at https:// dx.doi.org/10.21037/atm-21-4528
Conflicts of Interest: All authors have completed the ICMJE uniform disclosure form (available at https://dx.doi. org/10.21037/atm-21-4528). ZDH serves as the unpaid executive editor of the Annals of Translational Medicine from April 2014 to March 2025. Dr. GB reports support for attending meetings from BMS and Merck. The other authors have no conflicts of interest to declare.

Ethical Statement: The authors are accountable for all aspects of the work in ensuring that questions related to the accuracy or integrity of any part of the work are appropriately investigated and resolved.

Open Access Statement: This is an Open Access article distributed in accordance with the Creative Commons Attribution-NonCommercial-NoDerivs 4.0 International License (CC BY-NC-ND 4.0), which permits the noncommercial replication and distribution of the article with 
the strict proviso that no changes or edits are made and the original work is properly cited (including links to both the formal publication through the relevant DOI and the license). See: https://creativecommons.org/licenses/by-nc-nd/4.0/.

\section{References}

1. Buzby GP, Mullen JL, Matthews DC, et al. Prognostic nutritional index in gastrointestinal surgery. Am J Surg 1980;139:160-7.

2. Ignacio de Ulíbarri J, González-Madroño A, de Villar NG, et al. CONUT: a tool for controlling nutritional status. First validation in a hospital population. Nutr Hosp 2005;20:38-45.

3. Onodera T, Goseki N, Kosaki G. Prognostic nutritional index in gastrointestinal surgery of malnourished cancer patients. Nihon Geka Gakkai Zasshi 1984;85:1001-5.

4. Sun K, Chen S, Xu J, et al. The prognostic significance of the prognostic nutritional index in cancer: a systematic review and meta-analysis. J Cancer Res Clin Oncol 2014;140:1537-49.

5. Li D, Yuan X, Liu J, et al. Prognostic value of prognostic nutritional index in lung cancer: a meta-analysis. J Thorac Dis 2018;10:5298-307.

6. Wang Z, Wang Y, Zhang X, et al. Pretreatment prognostic nutritional index as a prognostic factor in lung cancer: Review and meta-analysis. Clin Chim Acta 2018;486:303-10.

7. Hu Y, Shen J, Liu R, et al. Prognostic value of pretreatment prognostic nutritional index in non-small cell lung cancer: A systematic review and meta-analysis. Int $\mathbf{J}$ Biol Markers 2018;33:372-8.

8. Jiang AM, Zhao R, Liu N, et al. The prognostic value of pretreatment prognostic nutritional index in patients with small cell lung cancer and it's influencing factors: a meta-analysis of observational studies. J Thorac Dis 2020;12:5718-28.

9. Zhang Z, Chen A, Xie F, et al. Low prognostic nutrition index predicts poorer quality of life in late-stage lung cancer. Ann Palliat Med 2020;9:3976-84.

10. Nakamura A, Kondo N, Nakamichi T, et al. Complications and Predictive Factors for Air Leak $>10$ Days with Neoadjuvant Chemotherapy Followed by Pleurectomy/ Decortication for Malignant Pleural Mesothelioma. Ann Surg Oncol 2021;28:3057-65.

11. Yao ZH, Tian GY, Wan YY, et al. Prognostic nutritional index predicts outcomes of malignant pleural mesothelioma. J Cancer Res Clin Oncol 2013;139:2117-23.
12. Tu X, Ren J, Zhao Y. Prognostic value of prognostic nutritional index in nasopharyngeal carcinoma: A meta-analysis containing 4511 patients. Oral Oncol 2020;110:104991.

13. Tang $M$, Jia $Z$, Zhang J. The prognostic role of prognostic nutritional index in nasopharyngeal carcinoma: A systematic review and meta-analysis. Int J Clin Oncol 2021;26:66-77.

14. Gao QL, Shi JG, Huang YD. Prognostic Significance of Pretreatment Prognostic Nutritional Index (PNI) in Patients with Nasopharyngeal Carcinoma: A MetaAnalysis. Nutr Cancer 2021;73:1657-67.

15. Bruixola G, Caballero J, Papaccio F, et al. Prognostic Nutritional Index as an independent prognostic factor in locoregionally advanced squamous cell head and neck cancer. ESMO Open 2018;3:e000425.

16. Kono T, Sakamoto K, Shinden S, et al. Pre-therapeutic nutritional assessment for predicting severe adverse events in patients with head and neck cancer treated by radiotherapy. Clin Nutr 2017;36:1681-5.

17. Yoshimura T, Suzuki H, Takayama H, et al. Impact of Preoperative Low Prognostic Nutritional Index and High Intramuscular Adipose Tissue Content on Outcomes of Patients with Oral Squamous Cell Carcinoma. Cancers (Basel) 2020;12:3167.

18. Zhao Y, Xu P, Kang H, et al. Prognostic nutritional index as a prognostic biomarker for survival in digestive system carcinomas. Oncotarget 2016;7:86573-83.

19. Xue Y, Zhou X, Xue L, et al. The role of pretreatment prognostic nutritional index in esophageal cancer: A metaanalysis. J Cell Physiol 2019;234:19655-62.

20. Liao G, Zhao Z, Yang H, et al. Can Prognostic Nutritional Index be a Prediction Factor in Esophageal Cancer?: A Meta-Analysis. Nutr Cancer 2020;72:187-93.

21. Hao J, Chen C, Wan F, et al. Prognostic Value of PreTreatment Prognostic Nutritional Index in Esophageal Cancer: A Systematic Review and Meta-Analysis. Front Oncol 2020;10:797.

22. Matsumoto Y, Zhou Q, Kamimura K, et al. The Prognostic Nutrition Index Predicts the Development of Hematological Toxicities in and the Prognosis of Esophageal Cancer Patients Treated with Cisplatin Plus 5-Fluorouracil Chemotherapy. Nutr Cancer 2018;70:447-52.

23. Li P, Wang X, Lai Y, et al. The prognostic value of pretreatment prognostic nutritional index in esophageal squamous cell carcinoma: A meta-analysis. Medicine (Baltimore) 2019;98:e15280. 
24. Chen S, Yang X, Feng JF. A novel inflammation-based prognostic score for patients with esophageal squamous cell carcinoma: the c-reactive protein/prognostic nutritional index ratio. Oncotarget 2016;7:62123-32.

25. Hirahara N, Tajima Y, Fujii Y, et al. Preoperative Prognostic Nutritional Index Predicts Long-Term Surgical Outcomes in Patients with Esophageal Squamous Cell Carcinoma. World J Surg 2018;42:2199-208.

26. Wang SH, Zhai ST, Lin H. Role of Prognostic Nutritional Index in patients with gastric cancer: a meta-analysis. Minerva Med 2016;107:322-7.

27. Yang Y, Gao P, Song Y, et al. The prognostic nutritional index is a predictive indicator of prognosis and postoperative complications in gastric cancer: A metaanalysis. Eur J Surg Oncol 2016;42:1176-82.

28. Li J, Xu R, Hu DM, et al. Prognostic Nutritional Index Predicts Outcomes of Patients after Gastrectomy for Cancer: A Systematic Review and Meta-Analysis of Nonrandomized Studies. Nutr Cancer 2019;71:557-68.

29. Yang Y, Gao P, Chen X, et al. Prognostic significance of preoperative prognostic nutritional index in colorectal cancer: results from a retrospective cohort study and a meta-analysis. Oncotarget 2016;7:58543-52.

30. Sun G, Li Y, Peng Y, et al. Impact of the preoperative prognostic nutritional index on postoperative and survival outcomes in colorectal cancer patients who underwent primary tumor resection: a systematic review and metaanalysis. Int J Colorectal Dis 2019;34:681-9.

31. Wang Z, Wang J, Wang P. The prognostic value of prognostic nutritional index in hepatocellular carcinoma patients: A meta-analysis of observational studies. PLoS One 2018;13:e0202987.

32. Man Z, Pang Q, Zhou L, et al. Prognostic significance of preoperative prognostic nutritional index in hepatocellular carcinoma: a meta-analysis. HPB (Oxford) 2018;20:888-95.

33. Fan X, Chen G, Li Y, et al. The Preoperative Prognostic Nutritional Index in Hepatocellular Carcinoma After Curative Hepatectomy: A Retrospective Cohort Study and Meta-Analysis. J Invest Surg 2021;34:826-33.

34. Liu J, Jiang S, Yang X, et al. The Significant Value of Preoperative Prognostic Nutritional Index for Survival in Pancreatic Cancers: A Meta-analysis. Pancreas 2018;47:793-9.

35. Li S, Tian G, Chen Z, et al. Prognostic Role of the Prognostic Nutritional Index in Pancreatic Cancer: A Meta-analysis. Nutr Cancer 2019;71:207-13.

36. Yamada S, Fujii T, Yabusaki N, et al. Clinical Implication of Inflammation-Based Prognostic Score in Pancreatic Cancer: Glasgow Prognostic Score Is the Most Reliable Parameter. Medicine (Baltimore) 2016;95:e3582.

37. Lv X, Zhang Z, Yuan W. Pretreatment Prognostic Nutritional Index (PNI) as a Prognostic Factor in Patients with Biliary Tract Cancer: A Meta-Analysis. Nutr Cancer 2021;73:1872-81.

38. Qi F, Zhou X, Wang Y, et al. Pre-treatment prognostic nutritional index may serve as a potential biomarker in urinary cancers: a systematic review and meta-analysis. Cancer Cell Int 2018;18:207.

39. Xiong SC, Hu X, Lia T, et al. Prognostic Significance of Prognostic Nutritional Index in Patients with Renal Cell Carcinoma: A Meta-Analysis. Nutr Cancer 2021. [Epub ahead of print].

40. Bi H, Shang Z, Jia C, et al. Predictive Values of Preoperative Prognostic Nutritional Index and Systemic Immune-Inflammation Index for Long-Term Survival in High-Risk Non-Muscle-Invasive Bladder Cancer Patients: A Single-Centre Retrospective Study. Cancer Manag Res 2020;12:9471-83

41. Karsiyakali N, Karabay E, Yucetas U. Predictive value of prognostic nutritional index on tumor stage in patients with primary bladder cancer. Arch Esp Urol 2020;73:132-9.

42. Yilmaz A, Y1lmaz H, Tekin SB, et al. The prognostic significance of hemoglobin-to-red cell distribution width ratio in muscle-invasive bladder cancer. Biomark Med 2020;14:727-38.

43. Shu K, Zheng Y, Chen J, et al. Prognostic value of selected preoperative inflammation-based scores in patients with high-risk localized prostate cancer who underwent radical prostatectomy. Onco Targets Ther 2018;11:4551-8.

44. Wang X, Wang Y. The prognostic nutritional index is prognostic factor of gynecological cancer: A systematic review and meta-analysis. Int J Surg 2019;67:79-86.

45. Dai Y, Liu M, Lei L, et al. Prognostic significance of preoperative prognostic nutritional index in ovarian cancer: A systematic review and meta-analysis. Medicine (Baltimore) 2020;99:e21840.

46. Kiuchi K, Hasegawa K, Ochiai S, et al. Prognostic significance of inflammatory parameters and nutritional index in clinical stage IVB endometrial carcinomas. J Obstet Gynaecol 2019;39:237-41.

47. Liu M, Wang L. Prognostic significance of preoperative serum albumin, albumin-to-globulin ratio, and prognostic nutritional index for patients with glioma: A meta-analysis. Medicine (Baltimore) 2020;99:e20927.

48. Mohri T, Mohri Y, Shigemori T, et al. Impact of 
prognostic nutritional index on long-term outcomes in patients with breast cancer. World J Surg Oncol 2016;14:170.

49. Yang Z, Zhang B, Hou L, et al. Pre-operative prognostic nutritional index predicts the outcomes for triple-negative breast cancer. Tumour Biol 2014;35:12165-71.

50. Wang Y, Battseren B, Yin W, et al. Predictive and prognostic value of prognostic nutritional index for locally advanced breast cancer. Gland Surg 2019;8:618-26.

51. Oba T, Maeno K, Ono M, et al. Prognostic Nutritional Index Is Superior to Neutrophil-to-lymphocyte Ratio as a Prognostic Marker in Metastatic Breast Cancer Patients Treated With Eribulin. Anticancer Res 2021;41:445-52.

52. Hua X, Long ZQ, Huang X, et al. The Value of Prognostic Nutritional Index (PNI) in Predicting Survival and Guiding Radiotherapy of Patients With T1-2N1 Breast Cancer. Front Oncol 2020;9:1562.

53. Oba T, Maeno K, Takekoshi D, et al. Neoadjuvant chemotherapy-induced decrease of prognostic nutrition index predicts poor prognosis in patients with breast cancer. BMC Cancer 2020;20:160.

54. Jiang K, Lei J, Li C, et al. Comparison of the prognostic values of selected inflammation based scores in patients with medullary thyroid carcinoma: A pilot study. J Surg Oncol 2017;116:281-7.

55. Offi C, Romano RM, Cangiano A, et al. Clinical significance of neutrophil-to-lymphocyte ratio, lymphocyte-to-monocyte ratio, platelet-to-lymphocyte ratio and prognostic nutritional index in low-risk differentiated thyroid carcinoma. Acta Otorhinolaryngol Ital 2021;41:31-8.

56. Chen W, Wei T, Li Z, et al. Association of the Preoperative Inflammation-Based Scores with TNM

Cite this article as: Yan L, Nakamura T, Casadei-Gardini A, Bruixola G, Huang YL, Hu ZD. Long-term and short-term prognostic value of the prognostic nutritional index in cancer: a narrative review. Ann Transl Med 2021;9(21):1630. doi: 10.21037/atm-21-4528
Stage and Recurrence in Patients with Papillary Thyroid Carcinoma: A Retrospective, Multicenter Analysis. Cancer Manag Res 2020;12:1809-18.

57. Lee SF, Ng TY, Wong FCS. The Value of Prognostic Nutritional Index in Follicular Lymphoma. Am J Clin Oncol 2019;42:202-7.

58. Chen KL, Liu YH, Li WY, et al. The prognostic nutritional index predicts survival for patients with extranodal natural killer/T cell lymphoma, nasal type. Ann Hematol 2015;94:1389-400.

59. Yao N, Hou Q, Zhang S, et al. Prognostic Nutritional Index, Another Prognostic Factor for Extranodal Natural Killer/T Cell Lymphoma, Nasal Type. Front Oncol 2020;10:877.

60. Paydas S, Lacin S, Dogan M, et al. Easier and more explanatory indices by integrating leukocyte lymphocyte ratio (LLR) and prognostic nutritional index (PNI) to IPS systems in cases with classical Hodgkin lymphoma. Leuk Res 2021;107:106586.

61. Mirili C, Paydas S, Kapukaya TK, et al. Systemic immuneinflammation index predicting survival outcome in patients with classical Hodgkin lymphoma. Biomark Med 2019;13:1565-75.

62. Matsukawa T, Suto K, Kanaya M, et al. Validation and comparison of prognostic values of GNRI, PNI, and CONUT in newly diagnosed diffuse large B cell lymphoma. Ann Hematol 2020;99:2859-68.

63. Luan C, Wang F, Wei N, et al. Prognostic nutritional index and the prognosis of diffuse large b-cell lymphoma: a meta-analysis. Cancer Cell Int 2020;20:455.

64. Matsuyama Y, Nakamura T, Yoshida K, et al. Role of the Prognostic Nutritional Index in Patients With Soft-tissue Sarcoma. In Vivo 2021;35:2349-55. 